\title{
POEMS syndrome presentation with progressive weakness in upper and lower limbs: A case report
}

\author{
CHUANG-JIE CAO ${ }^{*}$, CHENG-YUN DOU ${ }^{2 *}$, KE-HUA ZHOU $^{3}$, JIN-BO LIU $^{3}$ and HONG LAI ${ }^{3}$ \\ ${ }^{1}$ Department of Pathology, The First Affiliated Hospital of Sun Yat-san University, Guangzhou, Guangdong 510080; \\ Departments of ${ }^{2}$ Hepatology and ${ }^{3}$ Endocrinology, Qilu Hospital of Shandong University, Jinan, Shandong 250012, P.R. China
}

Received July 6, 2016; Accepted May 11, 2017

DOI: 10.3892/ol.2017.6904

\begin{abstract}
Polyneuropathy, organomegaly, endocrinopathy, $\mathrm{M}$ proteins, and skin changes (POEMS) syndrome is a rare variant of plasma cell disorders with multiple systemic manifestations. A 50-year-old female patient presented with progressive weakness in her upper and lower limbs; tingling, numbness and burning in her feet; polyneuropathy (demyelinating in the majority of cases of POEMS syndrome); monoclonal plasma cell disorder (typically $\lambda$-restricted in cases of POEMS syndrome); sclerotic lesions on the spine and pelvis; organomegaly, including hepatomegaly, splenomegaly and lymphadenopathy; edema; pleural effusion; adrenal, thyroidal, pituitary, gonadal and pancreatic endocrinopathy; skin changes, including hyperpigmentation, dry skin and hypertrichosis; thrombocytosis; pulmonary hypertension; low vitamin $\mathrm{B}_{12}$ and weight loss. Following the diagnosis of POEMS syndrome, the patient was treated only with pain-alleviating corticosteroids. Respiratory failure-induced mortality occurred 24 months after the patient first experienced difficulty walking and numbness in her lower extremities. The present study suggests that abnormal symptoms in cases of POEMS syndrome should be further evaluated during the diagnosis and treatment.
\end{abstract}

\section{Introduction}

Diagnosing polyneuropathy, organomegaly, endocrinopathy, and $\mathrm{M}$ protein and skin changes (POEMS) syndrome does

Correspondence to: Professor Hong Lai, Department of Endocrinology, Qilu Hospital of Shandong University, 107 Wenhuaxi Road, Jinan, Shandong 250012, P.R. China

E-mail: laihong@medmail.com.cn

${ }^{*}$ Contributed equally

Abbreviations: VEGF, vascular endothelial growth factor; PRL, prolactin; ACTH, adrenocorticotropic hormone; TEST, testosterone; IgG, immunoglobulin G; EMG, electromyography; MRI, magnetic resonance tomography; IL, interleukin

Key words: polyneuropathy, organomegaly, endocrinopathy, $\mathrm{M}$ proteins and skin changes syndrome, progressive weakness, diagnosis, anasarca, hyperpigmentation not require the patient to present all symptoms in the aforementioned acronym (1). Furthermore, the POEMS acronym does not include all POEMS syndrome-associated symptoms, and there is a Castleman disease variant of POEMS syndrome that may be associated with clonal plasma cell disorder (2). POEMS syndrome is a rare paraneoplastic syndrome associated with an underlying plasma cell disorder (2). Cases of POEMS syndrome have been reported in Japan (3-5), France, the United States, China and India (6-9). However, the incidence and prevalence of POEMS syndrome is unknown (10). Though the mechanism by which plasma cells cause POEMS syndrome remains to be fully understood, increased levels of vascular endothelial growth factor (VEGF) may be a factor (11).

POEMS syndrome is potentially fatal and is associated with a substantial deterioration in quality of life through neuropathy, anasarca, thromboembolic events and cachexia $(12,13)$. Early diagnosis and a multidisciplinary approach may increase the likelihood of reducing long-term irreversible morbidity.

Weakness in the upper and lower limbs is a common symptom of multiple diseases, including neuromuscular disorders (14), amyotrophic lateral sclerosis (15), multiple sclerosis, Guillain-Barre syndrome, transverse myelitis (16), hypovitaminosis D (17) and POEMS syndrome. Hence, presentation with weakness in the upper and lower limbs in patients with POEMS syndrome may not result in the correct diagnosis. The patient with POEMS syndrome described in the present study initially presented with weakness in the upper and lower limbs 2 years ago, and subsequently succumbed to respiratory failure at age 50 due to delayed diagnosis of POEMS syndrome. The present study intends to improve the understanding of POEMS syndrome among healthcare professionals.

\section{Case report}

On 3 March 2014, a 50-year-old female patient was admitted to the Department of Endocrinology, Qilu Hospital of Shandong University (Jinan, China), complaining of progressive weakness in her upper and lower limbs, which frequently failed. Furthermore, the patient was experiencing tingling, numbness and burning in her feet. The patient had a 3-year history of diffuse hyperpigmentation and slight anasarca, and a 2-month history of lumbodynia and debilitation, pain and numbness all over her body. In addition, the patient was diagnosed with 
type 2 diabetes at the Affiliated Hospital of Binzhou Medical School (Binzhou, China). Written informed consent was obtained from the patient for the publication of the present case report and any accompanying images.

On admission, a physical examination revealed chronic facies (pale or grey look caused by chronic disease) and unstable vital signs. Measurements were taken, including body temperature, $36.0^{\circ} \mathrm{C}$ (normal range, $36-37^{\circ} \mathrm{C}$ ); respiration rate, 19 breaths/min (normal range, 16-20 breaths/min); heart rate, 78 beats/min (normal range, $60-100$ beats/min); and blood pressure, 197/107 mmHg (normal range, $\leq 120 / 80 \mathrm{mmHg}$ ). Physical examination revealed diffuse hyperpigmentation, which was particularly severe on the upper trunk and limbs; dry skin; hypertrichosis (Fig. 1A); and leukonychia. The patient exhibited continued mild edema in her facial tissues and lower limbs (Fig. 1B). Palpable, soft rubbery lymph nodes were revealed in the axillary fossa. The patient exhibited splenomegaly and amenorrhea. Bilateral rales were heard with a stethoscope on auscultation. The body mass of the patient had decreased by $\sim 10 \mathrm{~kg}$ in the past year.

The blood cell count demonstrated an increase of platelets $\left(4.56 \times 10^{11}\right.$ cells/1), and a decrease in albumin $(29.3 \mathrm{~g} / \mathrm{l})$ and albumin/globulin ratio (0.88:1). However, electrolytes, liver and renal function were within normal limits. Analysis of blood coagulation serials revealed increased prothrombin time, increased standardization of prothrombin time ratio, decreased prothrombin time activity, activated partial thromboplastin time, increased plasma D-Dimer. These results revealed that the patient exhibited coagulation disorders. The results of endocrine hormone analysis revealed decreased free triiodothyronine, tetraiodothyronine and thyroglobulin, and increased thyroid-stimulating hormone, consistent with the symptoms of hypothyroidism. Symptoms of endocrinopathy included increased prolactin (PRL) and adrenocorticotropic hormone (ACTH), decreased testosterone (TEST), hypothyroidism, type 2 diabetes and empty sella turcica (Table I). Vitamin $B_{12}$ levels were $145.3 \mathrm{pg} / \mathrm{ml}$, which also decreased compared with the lower limits of the normal range (190-940 pg/ml).

An electromyography (EMG) test was used to identify neurological symptoms. The EMG test revealed motor and sensory polyneuropathy, with typical demyelination featuring at the proximal and distal ends of peripheral nerves in the four limbs of the patient. Administering hydrocortisone sodium succinate (100 mg/day) improved the neurological symptoms of the patient, though not markedly. An electrocardiogram revealed a low QRS voltage (limb leading) and a poor $\mathrm{R}$ wave progression. A chest radiograph demonstrated increased bronchovascular shadows, pachyntic pleurae and an enlarged, pear-shaped heart. An abdominal color Doppler ultrasonography scan revealed splenomegaly, pyoperitoneum and dropsy of the gallbladder wall. Echocardiographical analysis revealed enlarged atria, a dilatate main pulmonary artery, moderate mitral regurgitation, mild aortic regurgitation, severe tricuspid regurgitation, mild pulmonary regurgitation and severe pulmonary hypertension (Fig. 2). A computed tomography scan demonstrated that the lungs of the patient exhibited exudative alterations; scattered, and partial interstitial inflammation a thickened pulmonary artery; pulmonary hypertension; bilateral pleural; abdominal cavity and pericardial effusion; thoracic-abdominal wall and mesenterium edema. The patient also exhibited cholecystitis and multiple lymphadenopathy at heraxilla. Representative ascites, pleural and pericardial effusion and pulmonary edema were demonstrated in Fig. 3A pituitary gland magnetic resonance imaging (MRI) plain scan revealed partially empty sella. Plain computed tomography of lumbar vertebrae demonstrated a degenerative lumbar spine (calcification and small bony outgrowths), and L3/4 and L5/S1diskal hernia. MRI revealed osteosclerotic and blastic nodules and patches on the spine and pelvis.

In contrast with the hypoalbuminemia, immunoglobulin (Ig) $\mathrm{G}$, and $\lambda$ and $\kappa$ light chains were increased. Immunofixation electrophoresis was performed using the HYDRAGEL IF kit, according to the manufacturer's protocol (cat. no. PN4301; Sebia, Evry, France). The commercial HYDRAGEL IF (Sebia) was used, with a HYDRASYS 2 automated electrophoresis system (Sebia). The serum was diluted three times and the loading amount of each lane was $10 \mu \mathrm{l}$ and 2 repeats of serum protein electrophoresis with immunofixation were performed. For immunofixation, antibodies were provided by the HYDRAGEL commercial kit (cat. no. PN4301; Sebia) and were used as purchased. All durations and temperatures of incubations for electrophoresis with immunofixation were automated by HYDRASYS 2 (Sebia). The results of the present study demonstrated that $\gamma(\operatorname{IgG}), \kappa$ and $\mathrm{L}$ were positive, but $\alpha$ (IgA), $\mu(\operatorname{IgM}), \delta(\operatorname{IgD})$ were negative. The typical bands of protein electrophoresis are depicted in Fig. 4. Serological tests for tumor markers and rheumatism demonstrated negative results. Bone marrow puncture revealed reactive bone marrow and the presence of well-differentiated plasma cells.

POEMS syndrome was diagnosed using a combination of history and examination results according to the 2014 update on diagnosis, risk-stratification and management of POEMS syndrome (18). The presence of polyneuropathy (demyelinating in the majority of cases of POEMS syndrome); monoclonal plasma cell disorder (typically $\lambda$-restricted in cases of POEMS syndrome); sclerotic lesions on the spine and pelvis; organomegaly, including hepatomegaly, splenomegaly and lymphadenopathy; edema; pleural effusion; adrenal, thyroidal, pituitary, gonadal and pancreatic endocrinopathy; skin alterations, including hyperpigmentation, dry skin and hypertrichosis; thrombocytosis; pulmonary hypertension; low vitamin $B_{12}$ values; and weight loss supported the diagnosis of POEMS syndrome.

Subsequently, the patient received a low-dose steroid treatment (oral prednisolone $10 \mathrm{mg} /$ day). However, when the patient refused further positive medication, conservative treatment was used to alleviate the pain and relieve symptoms. Respiratory failure induced mortality in the patient 9 days following admission to the hospital; the patient was aged 50 .

\section{Discussion}

In 1980, Bardwick et al reported POEMS syndrome for the first time (19). Subsequently POEMS syndrome has been diagnosed based on a composite of clinical and laboratory features (12), and is misdiagnosed in patients that present with it if the syndrome is not considered. The pathogenesis of the syndrome remains to be fully understood. Overproduction of proinflammatory cytokines, including interleukin (IL)-1 $\beta$, IL-6, tumor necrosis factor- $\alpha$, and VEGF, have been reported 
Table I. Laboratory test results of the initial assessment of the patient.

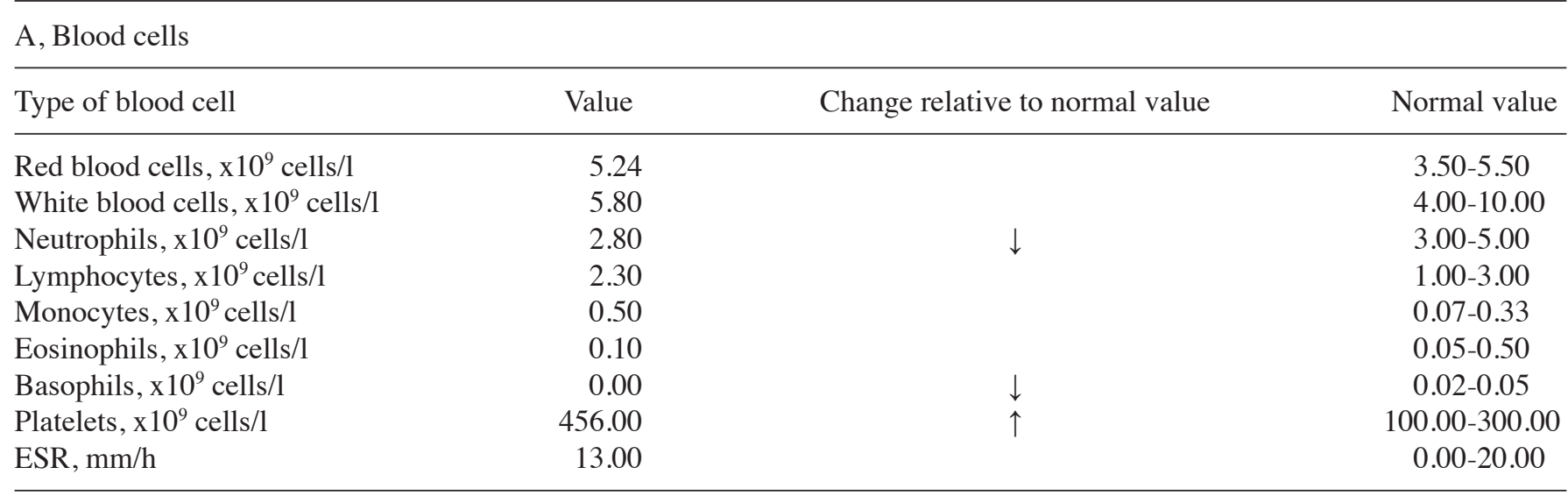

B, Proteins

\begin{tabular}{lccc}
\hline Type of protein & Concentration & Change relative to normal concentration & Normal concentration \\
\hline Albumin, g/l & 29.30 & $\downarrow$ & $40.00-55.00$ \\
Globulin, g/l & 33.20 & $\downarrow$ & $0.00-40.00$ \\
Albumin/globulin & $0.88: 1$ & & $1.20-2.40$ \\
Ig G, g/l & 15.00 & $\uparrow$ & $7.00-16.00$ \\
Ig A, g/l & 5.43 & & $0.70-4.00$ \\
Ig M, g/l & 0.88 & $\downarrow$ & $0.40-2.30$ \\
Ig E, g/l & 51.50 & & $0.00-100.00$ \\
C $3, g / 1$ & 0.56 & $\uparrow$ & $0.90-1.80$ \\
C4, g/l & 0.16 & $\uparrow$ & $0.10-0.40$ \\
$\kappa$ light chain, g/l & 4.18 & & $1.70-3.70$ \\
$\lambda$ light chain, g/l & 2.58 & & $0.90-2.10$ \\
$\kappa / \lambda$ & $1.62: 1$ & & $1.35-2.65$ \\
\hline
\end{tabular}

C, Coagulation serials

\begin{tabular}{lrcr}
\hline & Value & Change relative to normal value & Normal value \\
\hline Prothrombin time, sec & 21.10 & $\uparrow$ & $11.00-14.50$ \\
INR & 1.90 & $\uparrow$ & $0.80-1.20$ \\
Prothrombin time activity, $\%$ & 41.00 & $\downarrow$ & $70.00-120.00$ \\
APTT, sec & 86.30 & $\uparrow$ & $28.00-45.00$ \\
Fibrinogen, g/l & 2.11 & $\uparrow$ & $2.00-4.00$ \\
Plasma D-Dimer, $\mu \mathrm{g}$ & 3.77 & $\uparrow$ & $0.00-0.50$ \\
\hline
\end{tabular}

D, Hormone

\begin{tabular}{|c|c|c|c|}
\hline Type of hormone & Value & Change relative to normal value & Normal value \\
\hline Free triiodothyronine, $\mathrm{pmol} / \mathrm{l}$ & 2.21 & $\downarrow$ & $2.30-6.30$ \\
\hline Free tetraiodothyronine, $\mathrm{pmol} / \mathrm{l}$ & 6.28 & $\downarrow$ & $10.30-24.50$ \\
\hline Thyroid-stimulating hormone, UIU/ml & 6.19 & $\uparrow$ & $0.35-5.50$ \\
\hline Thyroglobulin, ng/ml & 0.32 & $\downarrow$ & $1.40-7.80$ \\
\hline Prolactin, ng/ml & 48.26 & $\uparrow$ & $3.40-24.10$ \\
\hline Testosterone, $\mathrm{ng} / \mathrm{ml}$ & $<0.03$ & $\downarrow$ & $0.06-0.82$ \\
\hline ACTH, pg/ml; 00:00 & 21.63 & $\uparrow$ & $0.00-10.00$ \\
\hline АCTH, pg/ml; 08:00 & 139.70 & $\uparrow$ & $4.70-48.80$ \\
\hline ACTH,pg/ml; 16:00 & 14.57 & & $0.00-46.00$ \\
\hline Vitamin $B_{12}, \mathrm{pg} / \mathrm{ml}$ & 145.30 & $\downarrow$ & $190.00-940.00$ \\
\hline
\end{tabular}

ESR, erythrocyte sedimentation rate; Ig, immunoglobulin; C, complement; INR, international normalized ratio; APTT, activated partial thromboplastin time; ACTH, adrenocorticotropic hormone. 

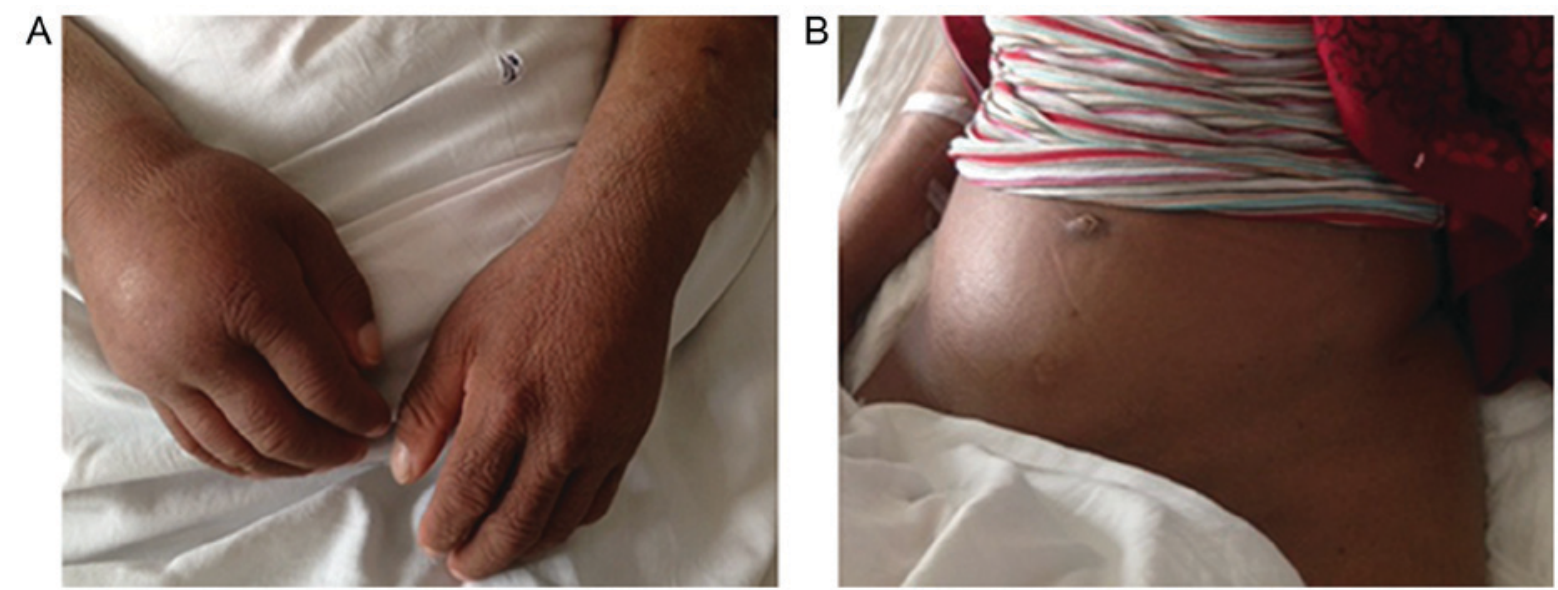

Figure 1. Photographs of the hands and abdomen of the patient. (A) Cutaneous hyperpigmentation and xerosis, and lipoatrophy and peripheral edema in the right hand of the patient. (B) Presentation of ascites and cutaneous hyperpigmentation.
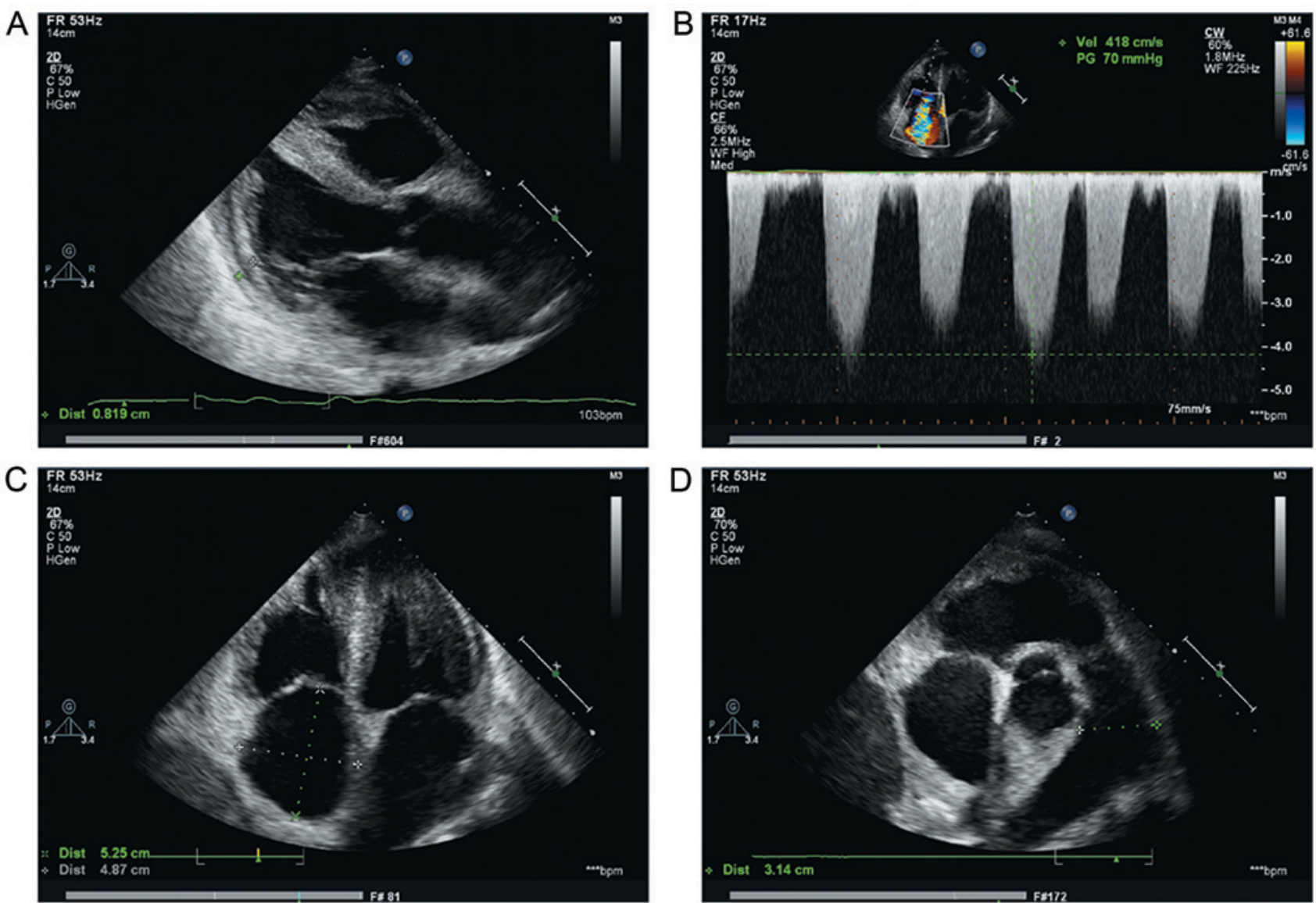

Figure 2. Results of echocardiographical analysis. (A) Thickness of the posterior wall of the left ventricle $(8.19 \mathrm{~mm})$. (B) Tricuspid regurgitation was detected in the right atrium during systolic; maximum pressure difference of tricuspid regurgitation $(\sim 70 \mathrm{mmHg})$, pulmonary artery systolic pressure $=85 \mathrm{mmHg}$. (C) Transverse diameter of the right atrium $(48.7 \times 52.5 \mathrm{~mm})$. (D) Internal diameter of the primary pulmonary artery $(31.4 \mathrm{~mm})$.

and may serve important etiological functions (20-22). Of these proinflammatory cytokines, only increased VEGF serves as a major criterion for the diagnosis of POEMS syndrome (12). VEGF potentially causes effusions, pulmonary hypertension and disseminated intravascular coagulation $(23,24)$, and is associated with POEMS syndrome activity (25).

The diagnosis of POEMS syndrome depends on a thorough history and a full examination of systems. The majority of authors agree that the presence of two major and at least one minor criteria are confirmatory (25). The mandatory major criteria for POEMS syndrome is polyradiculoneuropathy (typically demyelinating) and monoclonal plasma cell disorder. The patient described by the present study exhibited lumbodynia, pain and numbness all over the body, and the EMG test revealed extensive motor and sensory polyneuropathy, with typical demyelination at the proximal and distal 

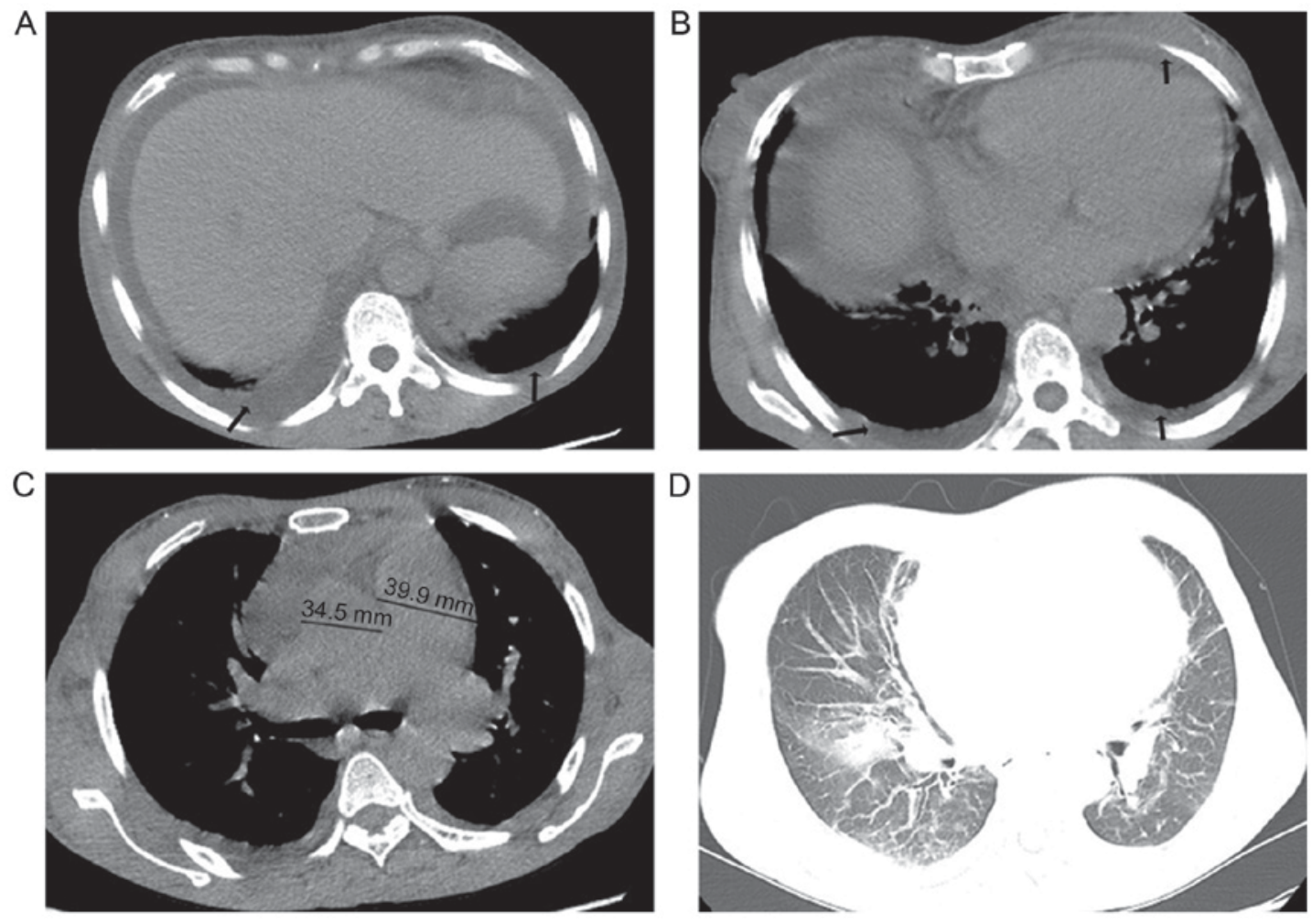

Figure 3. Representative CT images. (A) Arrows denote bilateral ascites. (B) Arrows denote bilateral pleural and pericardial effusion. (C) Pulmonary arterial hypertension (the diameter ratio of the pulmonary artery with aorta was $\geq 1$; the diameters of the pulmonary artery and the aorta were 39.9 and $34.5 \mathrm{~mm}$, respectively. (D) CT scan of the thorax revealed mixed ground-glass opacity and minimal consolidation, and intralobular reticulations and septal thickening, suggesting the presence of pulmonary edema. CT, computed tomography.

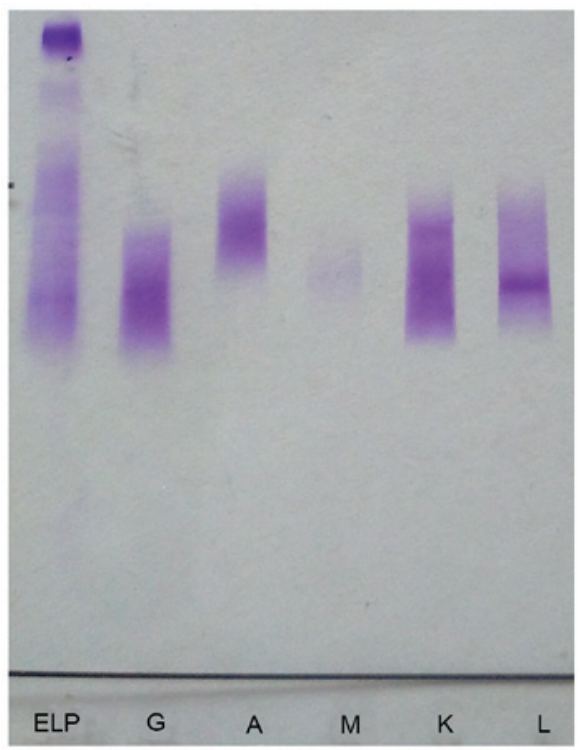

Figure 4. Serum protein electrophoresis with immunofixation revealed $\mathrm{IgG}$, $\kappa$ and $\mathrm{L}$ were positive, and that $\operatorname{IgA}$ and $\operatorname{IgM}$ were negative. $\mathrm{L}, \mathrm{K}, \mathrm{M}, \mathrm{A}, \mathrm{G}$ and ELP represent $\lambda$ light chain, $\kappa$ light chain, $\operatorname{IgM}, \operatorname{Ig} A, \operatorname{IgG}$ and reference light protein, respectively. Ig, immunoglobulin; ELP, elastin-like polypeptide.

ends of peripheral nerves in all four limbs. Serum-protein electrophoresis detected high levels of $\operatorname{IgG}, \lambda$ and $\kappa$ light chains; serum immunofixation demonstrated that $\operatorname{IgG}, \kappa$ and $\mathrm{L}$ were positive, and that $\operatorname{IgA}$ and $\operatorname{IgM}$ were negative, which matched with the results demonstrated by Dispenzieri et al (1). However, analysis of the bone marrow of the patient revealed no osteolytic lesions or plasma cell myeloma. MRI demonstrated a degenerative lumbar spine, L3/4 and L5/S1 diskal hernia and sclerotic bone lesion-associated osteosclerotic nodules on spine and pelvis. VEGF is associated with POEMS syndrome activity (24). However, since our hospital (Qilu Hospital of Shandong University) was not able to detect serum VEGF level, the present study did not determine the VEGF levels of the patient. In addition, organomegaly in POEMS syndrome typically occurs in the liver, spleen, and lymph nodes. The patient in the present study presented with splenomegaly and lymphadenopathy of bilateral axilla. Extravascular volume overload, including edema; pleural, abdominal cavity and pericardial effusion and ascites supported a diagnosis of POEMS syndrome. The presence of autoantibodies against the thyroid gland, type 2 diabetes, increased PRL and ACTH, decreasing TEST, and an empty sella turcica were considered to be evidence of endocrinopathy. Hyperpigmentation was the most common abnormality of skin alterations. A routine blood test identified thrombocytosis. Weight loss, pulmonary hypertension, thrombotic diatheses and low vitamin $\mathrm{B}_{12}$ levels also aided in the diagnosis of POEMS syndrome.

Currently, treatments for POEMS syndrome include radiation, chemotherapy, bone marrow transplantation and the use of other drugs, including alkylators, corticosteroids, bevacizumab, rituximab, bortezomib, and thalidomide $(26,27)$. Surgical resection maybe a potential treatment in the event of infection-associated complications, or the presence of a solitary tumor (28). Although an anti-VEGF strategy appears to be effective, limitations have been reported (29). Due to financial limitations and the severity of her condition, the patient in the 
present study refused any further positive therapy, using only pain-alleviating corticosteroids.

POEMS syndrome exhibits a chronic clinical course. Follow-up data revealed that the median survival time of patients is 165 months from the Mayo Clinic dysproteinmia database (1). A Chinese survey revealed that, 25 months on from the initial diagnosis, $80 \%$ of patients were alive after 25 months and $10 \%$ of these patients survived $>60$ months (8). Cardiorespiratory failure, along with pneumonia, was the most common means by which mortality occurred (1). Respiratory failure induced mortality in the patient described by the present study 24 months following the initial onset of difficulty walking and numbness in the lower limbs. The patient was aged 50.

POEMS syndrome is a rare paraneoplastic disorder consisting of peripheral neuropathy, organomegaly, endocrinopathy, and $\mathrm{M}$ protein and skin changes. The present study described a novel case of POEMS syndrome associated with the inconspicuous symptom of progressive weakness in the upper and lower limbs. The patient first entered hospital with the same symptom 2 years prior to her diagnosis. However, these symptoms were not identified as those of POEMS syndrome. Therefore, the present study recommends that more case reports concerning abnormal symptom-associated POEMS syndrome should be considered to improve the future diagnosis and treatment of POEMS syndrome, and enhance understanding of the syndrome.

\section{References}

1. Dispenzieri A, Kyle RA, Lacy MQ, Rajkumar SV, Therneau TM, Larson DR, Greipp PR, Witzig TE, Basu R, Suarez GA, et al: POEMS syndrome: Definitions and long-term outcome. Blood 101: 2496-2506, 2003.

2. Dispenzier A: POEMS syndrome: 2011 update on diagnosis, risk-stratification and management. Am J Hematol 86: 592-601, 2011.

3. Sanada S, Ookawara S, Karube H, Shindo T, Goto T, Nakamichi T, Saito M, Matsubara M and Suzuki M: Marked recovery of severe renal lesions in POEMS syndrome with high-dose melphalan therapy supported by autologous blood stem cell transplantation. Am J Kidney Dis 47: 672-679, 2006.

4. Nakamoto Y, Imai H, Yasuda T, Wakui H and Miura AB: A spectrum of clinicopathological features of nephropathy associated with POEMS syndrome. Nephrol Dial Transplant 14: 2370-2378, 1999.

5. Watanabe O, Arimura K, Kitajima I, Osame M and Maruyama I: Greatly raised vascular endothelial growth factor (VEGF) in POEMS syndrome. Lancet 347: 702, 1996.

6. Singh D, Wadhwa J, Kumar L, Raina V, Agarwal A and Kochupillai V: POEMS syndrome: Experience with fourteen cases. Leuk Lymphoma 44: 1749-1752, 2003.

7. Soubrier MJ, Dubost JJ and Sauvezie BJ: POEMS syndrome: A study of 25 cases and a review of the literature. French Study Group on POEMS syndrome. Am J Med 97: 543-553, 1994.

8. Li J, Zhou DB, Huang Z, Jiao L, Duan MH, Zhang W, Zhao YQ and Shen T: Clinical characteristics and long-term outcome of patients with POEMS syndrome in China. Ann Hematol 90: 819-826, 2011.

9. Kulkarni GB, Mahadevan A, Taly AB, Yasha TC, Seshagiri KS, Nalini A, Satishchandra P, Veerendrakumar M and Shankar SK: Clinicopathological profile of polyneuropathy, organomegaly, endocrinopathy, $\mathrm{M}$ protein and skin changes (POEMS) syndrome. J Clin Neurosci 18: 356-360, 2011

10. Plata Bello J and Garcia-Marin V: POEMS syndrome (polyneuropathy, organomegaly, endocrinopathy, multiple myeloma and skin changes) with cranial vault plasmocytoma and the role of surgery in its management: A case report. J Med Case Rep 7: $245,2013$.
11. Lee FY and Chiu HC: POEMS syndrome with calciphylaxis: A case report. Acta Derm Venereol 91: 96-97, 2011.

12. Dispenzieri A: POEMS syndrome: 2014 update on diagnosis, risk-stratification, and management. Am J Hematol 89: 214-223, 2014.

13. Nakamura Y, Nishimura M, Terano T, McNamara KM, Sasano H, Kurosu A and Joh K: A patient with POEMS syndrome: The pathology of glomerular microangiopathy. Tohoku J Exp Med 231: 229-234, 2013.

14. Kaya P, Alemdaroğlu I, Yilmaz Ö, Karaduman A and Topaloğlu H: Effect of muscle weakness distribution on balance in neuromuscular disease. Pediatr Int 57: 92-97, 2015.

15. Devine MS, Kiernan MC, Heggie S, McCombe PA and Henderson RD: Study of motor asymmetry in ALS indicates an effect of limb dominance on onset and spread of weakness and an important role for upper motor neurons. Amyotroph Lateral Scler Frontotemporal Degener 15: 481-487, 2014.

16. Kuo DC, Bilal S and Koller P: Asymmetric weakness and west nile virus infection. J Emerg Med 49: 274-276, 2015.

17. Iolascon G, de Sire A, Calafiore D, Moretti A, Gimigliano R and Gimigliano F: Hypovitaminosis D is associated with a reduction in upper and lower limb muscle strength and physical performance in post-menopausal women: A retrospective study. Aging Clin Exp Res 27 (Suppl 1): S23-S30, 2015.

18. Dispenzieri A: POEMS Syndrome: 2014 update on diagnosis, risk-stratification and management. Am J Hematol 89: 213-223, 2014.

19. Bardwick NJ, Zvaifler GN, Gill GN, Newman D, Greenway G and Resnick DL: Plasma cell dyscrasia with polyneuropathy, organomegaly, endocrinopathy, $\mathrm{M}$ protein, and skin changes: The POEMS syndrome. Report on two cases and a review of the literature. Medicine (Baltimore) 59: 311-322, 1980.

20. Rose C, Zandecki M, Copin MC, Gosset P, Labalette M, Hatron PY, Jauberteau MO, Devulder B, Bauters F and Facon T: POEMS syndrome: Report on six patients with unusual clinical signs, elevated levels of cytokines, macrophage involvement and chromosomal aberrations of bone marrow plasma cells. Leukemia 11: 1318-1323, 1997.

21. Hitoshi S, Suzuki K and Sakuta M: Elevated serum interleukin-6 in POEMS syndrome reflects the activity of the disease. Intern Med 33: 583-587, 1994.

22. Soubrier M, Sauron C, Souweine B, Larroche C, Wechsler B, Guillevin L, Piette JC, Rousset H and Deteix P: Growth factors and proinflammatory cytokines in the renal involvement of POEMS syndrome. Am J Kidney Dis 34: 633-638, 1999.

23. Tokashiki T, Hashiguchi T, Arimura K, Eiraku N, Maruyama I and Osame M: Predictive value of serial platelet count and VEGF determination for the management of DIC in the Crow-Fukase (POEMS) syndrome. Intern Med 42: 1240-1243, 2003.

24. Niimi H, Arimura K, Jonosono M, Hashiguchi T, Kawabata M, Osame $\mathrm{M}$ and Kitajima I: VEGF is causative for pulmonary hypertension in a patient with Crow-Fukase (POEMS) syndrome. Intern Med 39: 1101-1104, 2000.

25. Dispenzieri A: How I treat POEMS syndrome. Blood 119: 5650-5658, 2012.

26. Kawano Y,Nakama T, Hata H, Kimura E, Maruyoshi N, Uchino M and Mitsuya H: Successful treatment with rituximab and thalidomide of POEMS syndrome associated with Waldenstrom macroglobulinemia. J Neurol Sci 297: 101-104, 2012.

27. Ohwada C, Nakaseko C, Sakai S, Takeda Y, Abe D, Takeuchi M, Sakaida E, Masuda S, Shimizu N, Cho R, et al: Successful combination treatment with bevacizumab, thalidomide and autologous PBSC for severe POEMS syndrome. Bone Marrow Transplant 43: 739-740, 2009.

28. Kotani Y, Ito M, Abumi K, Yasui K and Minami A: Neurological recovery from pentaplegia after resection for sacral plasmacytoma presenting with POEMS syndrome. J Neurosurg Spine 10: 324-328, 2009.

29. Allam JS, Kennedy CC, Aksamit TR and Dispenzieri A: Pulmonary manifestations in patients with POEMS syndrome: A retrospective review of 137 patients. Chest 133: 969-974, 2008. 